\title{
The abundance of the spliceosomal snRNPs is not limiting the splicing of U12-type introns
}

\author{
HELI K.J. PESSA, ANNUKKA RUOKOLAINEN, and MIKKO J. FRILANDER \\ Institute of Biotechnology, Program on Developmental Biology, PL56 (Viikinkaari 9), 00014 University of Helsinki, Finland
}

\begin{abstract}
The rate of excision of U12-type introns has been reported to be slower than that of U2-type introns, suggesting a rate-limiting bottleneck that could down-regulate genes containing U12-type introns. The mechanistic reasons for this slower rate of intron excision are not known, but lower abundance of the U12-type snRNPs and slower rate of assembly or catalytic activity have been suggested. To investigate snRNP abundance we concentrated on the U4atac snRNA, which is the least abundant of the U12-type snRNAs and is limiting the formation of U4atac/U6atac complex. We identified mouse NIH-3T3 cell line isolates in which the level of both U4atac snRNA and U4atac/U6atac complexes is reduced to $10 \%-20 \%$ of the normal level. We used these cell lines to investigate splicing efficiency by transient transfection of a reporter gene containing a U12-type intron and by quantitative PCR analysis of endogenous genes. The splicing of the reporter U12-type intron was very inefficient, but the activity could be restored by overexpression of U4atac snRNA. Using these U4atac-deficient NIH-3T3 cells, we confirmed the results of previous studies showing that U12-type introns of endogenous genes are, indeed, excised more slowly than U2-type introns, but we found that the rate did not differ from that measured in cells displaying normal levels of U4atac snRNA. Thus our results suggest that the cellular abundance of the snRNPs does not limit U12-type intron splicing under normal conditions.
\end{abstract}

Keywords: U12-type introns; U4atac snRNA; spliceosome; splicing rate

\section{INTRODUCTION}

U12-type introns have been identified in the genomes of many metazoans, including vertebrates, plants, and insects (Levine and Durbin 2001; Zhu and Brendel 2003; Schneider et al. 2004), but they are absent, for example, from Caenorhabditis elegans and unicellular organisms (Burge et al. 1998). Compared with the more common U2-type introns, the U12type introns contain unusually conserved $5^{\prime}$ splice-site ( $\left.5^{\prime} \mathrm{ss}\right)$ and branch-point sequences (BPS), which can be identified by bioinformatic analyses (Burge et al. 1998, 1999; Abril et al. 2005). In vertebrates, U12-type introns comprise $\sim 0.35 \%$ of all introns. The human genome contains $\sim 400$ genes with U12-type introns (Levine and Durbin 2001), and the number is expected to be similar in other mammals and possibly in birds (chicken) as well (Abril et al. 2005); the reported numbers in the Arabidopsis and Drosophila genomes are 165 and 20, respectively (Zhu and Brendel 2003; Schneider et al.

Reprint requests to: Mikko J. Frilander, Institute of Biotechnology, Program on Developmental Biology, PL56 (Viikinkaari 9), 00014 University of Helsinki, Finland; e-mail: Mikko.Frilander@Helsinki.Fi; Fax. +358-9-191 59366.

Article published online ahead of print. Article and publication date are at http://www.rnajournal.org/cgi/doi/10.1261/rna.213906.
2004). U12-type introns are concentrated in so-called information processing genes, which include such functions as DNA replication and repair, gene expression (transcription, RNA processing and transport, translation), and many functions related to cytoskeletal organization; they are virtually absent, however, from genes involved in metabolic pathways (Burge et al. 1998; Levine and Durbin 2001).

The U12-type introns are removed by the dedicated U12-dependent spliceosome, whereas the more abundant U2-type introns are excised by the U2-dependent spliceosome. Both spliceosomes have similar assembly pathways and catalytic mechanisms (Patel and Steitz 2003; Will and Lührmann 2005), and both are composed of five small nuclear RNAs (snRNAs) and numerous protein factors. The U1, U2, U4, and U6 snRNAs are specific to the U2-dependent spliceosome, whereas the U12-dependent spliceosome uses the functional analogs U11, U12, U4atac, and U6atac, respectively (Hall and Padgett 1996; Tarn and Steitz 1996a, b; Kolossova and Padgett 1997; Incorvaia and Padgett 1998; Shukla and Padgett 1999). The U5 snRNA functions in both spliceosomes (Tarn and Steitz 1997). Moreover, most of the spliceosomal protein components appear to be shared between the two spliceosomes (Will and Lührmann 2005)—only seven proteins have been 
identified that are specific to the U12-dependent spliceosome. All unique proteins are found in the U11/U12 di-snRNP complex, suggesting a possible role in intron recognition (Will et al. 1999, 2004). In contrast, the protein composition of the U4atac/U6atac.U5 tri-snRNP is most likely similar or identical to that of the U2-dependent spliceosome (Schneider et al. 2002). Consistent with this hypothesis, it has been found that U4 snRNA can function in the U12-dependent spliceosome if the necessary basepairing interactions with U6atac snRNA are provided (Shukla and Padgett 2004), suggesting that there are no U4atac-specific protein components in the U12-dependent spliceosome.

The relatively slow splicing kinetics of U12-type introns observed in vitro (Tarn and Steitz 1996b; Wu and Krainer 1996) and in vivo (Patel et al. 2002) has led to the suggestion that the processing of U12-type introns could be rate limiting for the expression of genes containing such introns. This assertion is supported by the study of Patel et al. (2002), which showed that conversion of a U12-type intron to a normal U2-type intron in a reporter gene increased the reporter expression by up to 10 -fold. These results suggest the possibility that U12-type introns may indeed provide a post-transcriptional mechanism by which the expression of certain genes could be limited. Additionally, a slower excision rate for a U12-type intron in vivo has been reported earlier with the RPL1 gene in Xenopus oocytes (Santoro et al. 1994). However, the mechanistic basis for the slower intron removal is not known. One possibility is that gene down-regulation is achieved via the lower abundance of snRNAs associated with the U12-dependent spliceosome or through specific protein components.

In mammalian cells, the abundance of the snRNP components of the U12-dependent spliceosome is $\sim 2 \times$ $10^{3}$ to $10^{4}$ particles per cell, which is $\sim 100$-fold less than that of the U2-dependent spliceosome counterparts (Montzka and Steitz 1988; Tarn and Steitz 1996a; Yu et al. 1999). However, it is not clear whether this reduced number of snRNPs slows splicing or spliceosome assembly in vivo, because the number of genes containing U12-type introns in mammalian cells is also relatively small-400 such genes have been detected in humans (Levine and Durbin 2001). Other factors that could affect the splicing efficiency of the U12-type introns are the specific proteins of the U11/U12 di-snRNP, which could affect either intron recognition or later assembly steps. In contrast, tri-snRNPspecific protein components are shared between the two spliceosomes and thus are unlikely to regulate spliceosomespecific splicing (Schneider et al. 2002).

In this study we have identified mouse cell lines 3T3-D1 and 3T3-D2, derived from the commonly used NIH-3T3 line, that display significantly reduced expression of the U4atac snRNA as compared with other snRNAs specific to either U2- or U12-dependent spliceosomes. This reduction causes an up to 10-fold decrease in the amount of the
U4atac/U6atac complex in the cell. We tested the splicing using a reporter construct that contains a U12-type intron and that was transiently overexpressed in the cells. Additionally, we measured the splicing activity of several endogenous genes containing U12-type introns. We found very inefficient excision of the U12-type intron in 3T3-D1 cells when the reporter construct was overexpressed alone. The splicing was restored to control cell line (L-929) levels by coexpression of U4atac snRNA. Therefore, the reduced efficiency of splicing with the reporter construct was most likely due to the lower abundance of U4atac snRNA. In contrast, excision of the U12-type introns in endogenous genes was not affected by the low U4atac levels, but was equally efficient in our 3T3-D1 and L-929 cells. Thus our results suggest that cells contain a relative surplus of U12type snRNPs, and their number is not limiting under normal conditions.

\section{RESULTS}

\section{Identification of NIH-3T3 isolates containing significantly reduced amounts of U4atac snRNA and U4atac/U6atac complex}

We used Northern blotting to analyze the abundance of U12-dependent spliceosomal components in various mouse and human cell lines. Variations among cell types in this regard would suggest that the relevant components may serve to regulate the activity of the U12-dependent spliceosome. We identified two mouse NIH-3T3 cell isolates (3T3D1 and 3T3-D2) in which the level of U4atac snRNA was significantly reduced compared with other cell lines (e.g., mouse L-929 cells or HeLa cells). Comparison of these NIH3T3 cells with L-929 and HeLa cells (Fig. 1A) showed little variation in the levels of $\mathrm{U} 2$-dependent spliceosome-specific snRNAs (U1, U2, U4, U5, U6) or U11, U12 snRNAs of the U12-dependent spliceosome. However, the level of U4atac snRNA in both NIH-3T3 cell line isolates (Fig. 1A, lanes 2,3) was reduced by $80 \%$ relative to control cells. The relative levels of U4atac and the other snRNAs detected in both human and mouse control cell lines were similar to those in most mouse tissues (data not shown). The 3T3-D2 line also had a reduced level of U6atac snRNA (Fig. 1A, lane 3); therefore, to simplify the subsequent functional analysis, we used only the 3T3-D1 line.

We prepared nuclear extracts from HeLa, L-929, and 3T3-D1 cells to assess whether U4atac/U6atac complex formation was affected in the 3T3-D1 cells. The extracts were then treated with proteinase $\mathrm{K}$ at low temperature conditions to maintain the base pairing between U4atac and U6atac snRNAs. Half of each sample was loaded directly into a native gel (to identify snRNA complexes), whereas the other half was heat denatured prior to loading (to identify free snRNAs). Quantitation of Northern blots (normalized to U12) showed that the low level of U4atac 
A

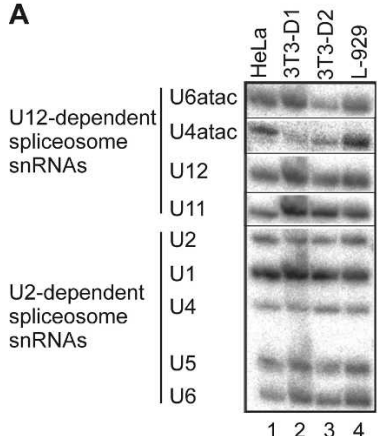

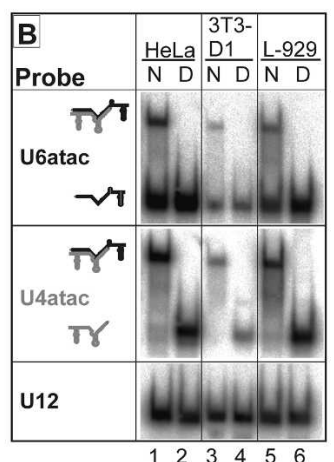

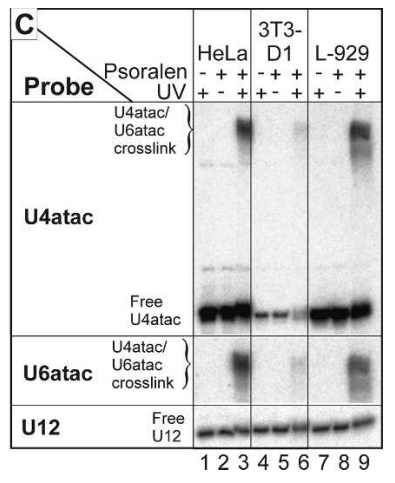

FIGURE 1. (A) Comparison of the expression levels of spliceosomal snRNAs in HeLa cells, two independent isolates of NIH-3T3 cells (3T3-D1 and 3T3-D2), and L-929 cells. Total RNA was extracted from each cell line, separated by denaturing polyacrylamide gel electrophoresis, and blotted to a nylon filter. Individual snRNAs were detected by Northern blotting. $(B)$ U4atac/U6atac complex formation in HeLa, 3T3-D1, and L-929 cells. RNAs were isolated from nuclear extracts by proteinase $\mathrm{K}$ treatment under mild conditions to preserve U4atac/U6atac base pairing. Subsequently, the isolated RNA preparations were separated in native gels and transferred to nylon filters. Half of each sample was kept under native $(\mathrm{N})$ conditions prior to gel loading. The other half (D) was heat denatured at $95^{\circ} \mathrm{C}$ for $3 \mathrm{~min}$ (to break the U4atac/ U6atac base pairing) followed by snap cooling on wet ice prior to loading. U4atac, U6atac, and U12 snRNAs were detected by Northern hybridization. The migration of individual U4atac (gray) and U6atac (black) snRNAs and the U4atac/U6atac complex is indicated on the left. $(C)$ Detection of the U4atac/U6atac interaction by psoralen cross-linking. Nuclear extracts from HeLa, 3T3-D1, and L-929 cells were treated with AMT-psoralen, UV light, or both as indicated above each lane. Following deproteinization with proteinase $\mathrm{K}$ the samples were separated in denaturing $6 \%$ polyacrylamide gels and analyzed by Northern hybridization as in $B$.

of the mouse $s m E$ gene. The human ortholog of $s m E$ has been used previously to measure the efficiency of the U12-dependent spliceosome (Patel et al. 2002). A region covering exons 1 through 3 of mouse $s m E$ was fused in frame with the CFP portion of the Drosophila reporter gene, including the relatively small U2-type intron from the Drosophila construct, which we found to be spliced efficiently and accurately in mouse cells (data not shown). Thus, in this reporter construct the first intron was of the U12-type and the others were of the U2-type (Fig. 2A). Additionally, we created a U4atac snRNA expression construct, in which the native U4atac promoter was changed to the promoter from the mouse U12 snRNA gene to ensure efficient expression of U4atac snRNA in 3T3-D1 cells.

We transiently transfected the reporter construct into 3T3-D1 cells and L-929 cells (which have normal levels of U4atac) and looked for differences in splicing of the U12-type intron. Northern blot quantification of the RNA levels

snRNA in 3T3-D1 cells indeed reduced U4atac/U6atac complex formation by $90 \%$ relative to control cells (see Fig. 1B, cf. lane 3 and lanes 1,5). A similar reduction was detected when the 3T3-D1 nuclear extract was subjected to cross-linking using AMT-psoralen (Frilander and Steitz 2001) to identify the base-paired U4atac/U6atac complexes (Fig. 1C, cf. lane 6 and lanes 3,9). With nuclear extract preparations we also detected a reduced level of free U6atac snRNA (Fig. 1B, lanes 4 and 6) in 3T3-D1 cells compared with the control cells, even though total RNA preparations from the same cells (see Fig. 1A) indicated that this should not be the case. However, this reduction in U6atac snRNA was not detected when the U4atac/U6atac complexes were analyzed in whole-cell lysates (see Fig. 3), suggesting that this phenomenon is specific to nuclear extract preparations from the 3T3-D1 cells.

\section{Low U4atac levels inhibit U12-dependent splicing in vivo}

To test whether relatively low levels of U4atac snRNA affect splicing in vivo, we constructed a reporter plasmid containing a U12-type intron. Initially we tested the cyan fluorescent protein (CFP) reporter plasmid based on the Drosophila gene, NHE3 (Patel et al. 2002), but found that the first (U2-type) intron of this construct was not spliced in the mammalian system (data not shown). Therefore, we replaced the NHE3 part of the reporter gene with a portion of the reporter gene (taking into account the low $\sim 10 \%$ transfection efficiency, which is characteristic with both cell lines) indicated at least 50 -fold overexpression compared to the endogenous $s m E$ locus in both cell lines (data not shown). Northern blotting revealed that splicing was very efficient in L-929 cells (Fig. 2B, lane 8), whereas transfection into 3T3-D1 cells yielded a diffuse band for the spliced mRNA in which the majority of the mRNA was in a slowly migrating species at the position expected for the unspliced reporter (Fig. 2B, cf. lanes 1 and 8). An intron-specific probe indicated that this band contained the U12-type intron of the reporter construct (not shown). The diffuse appearance of the unspliced mRNA band was most likely due to degradation similar to that observed by Patel et al. (2002). The accumulation of the unspliced U12-type intron was confirmed by RT-PCR analysis of the same samples using primers specific to exon 1 of the mouse $s m E$ gene and the CFP parts of the reporter construct. In 3T3-D1 cells, two main PCR bands were detected: a major 850-bp band and a weaker 250-bp band representing unspliced and spliced mRNAs, respectively (Fig. 2C, lane 1). In contrast, in L-929 cells the spliced 250-bp band predominated, whereas the unspliced 850-bp band was almost undetectable (Fig. 2C, lane 4). The identities of the PCR bands were confirmed by cloning and sequencing, which revealed that the 850-bp PCR product contained an unspliced U12-type intron and correctly spliced U2-type introns while the 250-bp product corresponded to the spliced reporter mRNA from which 

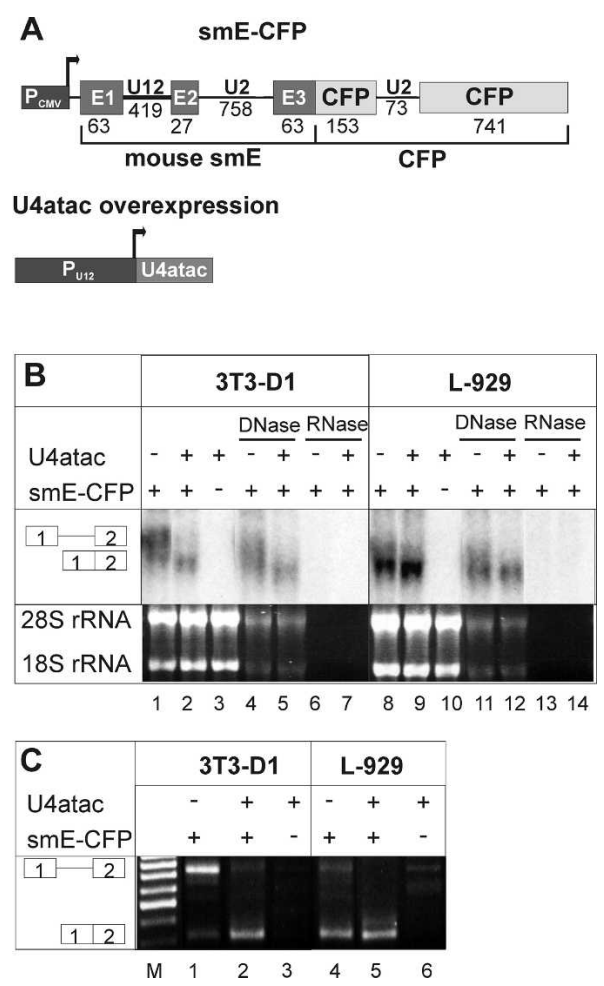

FIGURE 2. (A) Schematic diagrams of the smE-CFP (splicing reporter) and the U4atac overexpression constructs. (Top) The smECFP construct is controlled by the CMV promoter and contains a portion of mouse $s m E$ (exons 1-3) fused to the cyan fluorescent protein (CFP) portion of the reporter construct described by Patel et al. (2002). The lengths of the exons and introns and the intron type (U12- or U2-type) are indicated. (Bottom) Schematic diagram of the U4atac overexpression construct. The coding sequence of the U4atac snRNA gene and $3^{\prime}$ box downstream of the coding region were fused with the U12 snRNA promoter. In both constructs the transcription start site is indicated by an arrow. (B) Effect of U4atac overexpression on splicing in 3T3-D1 and L-929 cells. Total RNA $(8.5 \mu \mathrm{g})$ from transiently transfected 3T3-D1 (lanes 1-3) and L-929 (lanes 8-10) cells was analyzed by Northern blotting using a probe specific to smE-CFP exons. DNase treated lanes $(4,5$ and 11,12$)$ contained approximately $4 \mu \mathrm{g}$ of total RNA. Cells were transfected with smECFP alone, with U4atac alone, or with both constructs as indicated above each lane. In lanes 4-7 and 11-14, the samples were treated with either DNase or RNase as indicated, to confirm that the observed signal was derived from RNA. The migration of the reporter transcript with either unspliced or spliced U12-type intron 1 is indicated on the left. (Bottom) Ethidium bromide-stained gel from the same experiment showing $28 \mathrm{~S}$ and $18 \mathrm{~S}$ rRNAs as a loading control. (C) RT-PCR analysis of the effect of U4atac snRNA overexpression in 3T3-D1 and L-929 cells. The migration of the PCR products with either unspliced or spliced U12-type intron 1 is indicated on the left.

each of the three introns, including the U12-type intron, were correctly removed. Thus, both the Northern blotting and RT-PCR results indicated that the removal of the U12type intron of the reporter construct was inefficient in 3T3D1 cells.

To test if the observed effects were indeed due to the reduced amounts of U4atac snRNA in 3T3-D1 cells, we transiently cotransfected the U4atac snRNA expression plasmid together with reporter construct (Fig. 2A) into 3T3-D1 and L-929 cells. Since the U4atac snRNP does not contain protein components specific to the U12-dependent spliceosome (Schneider et al. 2002; Shukla and Padgett 2004), we expected that overexpression of the U4atac snRNA alone would be sufficient to increase the level of U4atac/U6atac complexes (or the U4atac/U6atac.U5 tri-snRNP). Consistent with this supposition, native gel analysis of the base-paired U4atac/U6atac snRNA complexes from stably transfected cell lines indeed indicated that U4atac snRNA overexpression in 3T3-D1 cells increased the number of U4atac/U6atac complexes to a level similar to that in L-929 cells (Fig. 3). In L-929 cells, U4atac snRNA overexpression resulted in a large increase in the U4atac snRNA level, as illustrated by increases in the levels of both the free U4atac snRNA and the U4atac/ U6atac complex (Fig. 3, lane 5).

Northern blot analysis of the processing of smE-CFP in 3T3-D1 cells that were transiently transfected with both the reporter construct and U4atac expression plasmid revealed that coexpression of U4atac snRNA significantly reduced the level of the slowly migrating unspliced pre-mRNA in the transfected cells and increased the intensity of the mRNA band (Fig. 2B, cf. lanes 1 and 2). These results were supported by the RT-PCR analysis, which also showed that coexpression of U4atac snRNA increased the level of the

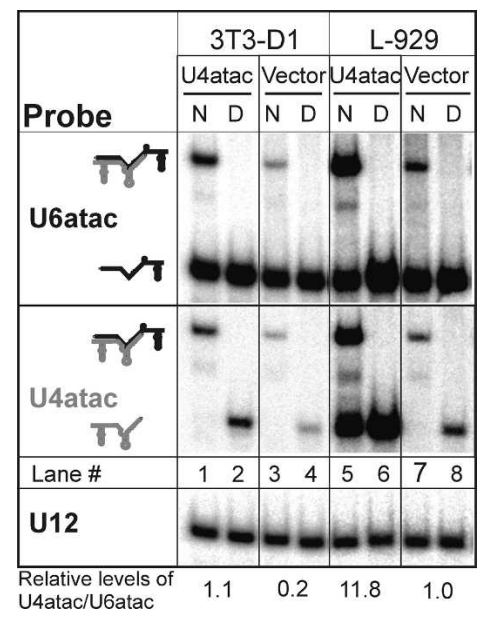

FIGURE 3. The effect of stable U4atac overexpression on U4atac/ U6atac complex formation in 3T3-D1 and L-929 cells. U4atac/U6atac complexes were isolated and analyzed as in Figure 1B, except that whole-cell lysates were used instead of nuclear extracts. Complexes were analyzed from stable U4atac overexpression lines (U4atac) or from cell lines transfected with the expression vector (vector) without the U4atac expression cassette. The bottom of the figure shows the relative levels of the U4atac/U6atac complex, as determined by phosphorimager analysis of the blots probed for U12, U4atac, and U6atac snRNAs. The intensities of the U4atac/U6atac bands were determined from native lanes $(1,3,5$, and 7$)$ and the mean value from the blots probed for U4atac and U6atac was used to calculate the relative levels of U4atac/U6atac complexes. The raw data were first normalized against the level of U12 snRNA signal and then scaled such that the value in L-929 cells in lane 7 was set to 1.0. The labels and symbols are as in Figure $1 \mathrm{~B}$. 
fully spliced 250-bp band and decreased that of the partially spliced 850-bp band in 3T3-D1 cells (Fig. 2C, lanes 1 and 2). With L-929 cells, we detected a slight increase in the mRNA signal in the Northern blot (Fig. 2B, cf. lanes 8 and 9) and in the RT-PCR analysis (Fig. 2C, lanes 4 and 5). Additionally, the signal from the partially spliced 850-bp band disappeared almost completely in L-929 cells upon U4atac overexpression (Fig. 2C, lanes 4 and 5). Thus, together these results suggest that the reduced level of U4atac snRNA leads to inefficient splicing of the reporter construct. The effect appears to be specific for U4atac reduction, since the splicing efficiency was restored to normal levels upon overexpression of U4atac snRNA in 3T3-D1 cells. We therefore conclude that a reduction of the spliceosome abundance can affect the splicing of a reporter construct under overexpression conditions.

\section{U12-type introns in endogenous genes are spliced efficiently in 3T3-D1 cells}

We next asked whether the inefficient splicing observed with the U12-type intron-containing reporter construct could also be detected with endogenous genes. Based on an existing list of human U12-type intron-containing genes (Levine and Durbin 2001), we selected mouse orthologs of six human genes, namely Pex16, Drap1, Ipo4, Psmc4, smE, and Gars, each carrying a U12-type intron with 5 ss and BPS sequences that closely resemble the known consensus sequences (see Fig. 4). Comparison of the selected mouse genes to orthologs in other mammalian and vertebrate genomes (available at www.ensembl.org) indicated the presence of homologous U12-type introns with well-conserved 5 'ss and BPS sequences in all mammalian and most other vertebrate genomes (data not shown). This phylogenetic conservation further suggested that these orthologs contained authentic U12-type introns. With Ipo4 the proper formation of U12-type spliceosomal complexes and their functionality in in vitro splicing have also been tested in HeLa nuclear extract (J. Turunen and M. Frilander, unpubl. data).

The splicing efficiency of U12-type introns was tested by quantitative RT-

Importin 4 (/po4)

B
PCR (qPCR) analysis of total RNA samples isolated from nontransfected 3T3-D1 and L-929 cells and from the same cell lines that had been stably transfected with the U4atac expression plasmid. U4atac overexpression increased the level of the U4atac/U6atac complex in 3T3-D1 cells to that observed in untransfected L-929 cells (see Fig. 3). We used intron-specific and exon-spanning qPCR primers to measure the abundance of pre-mRNAs/mRNAs in which the intron under investigation has been either retained or
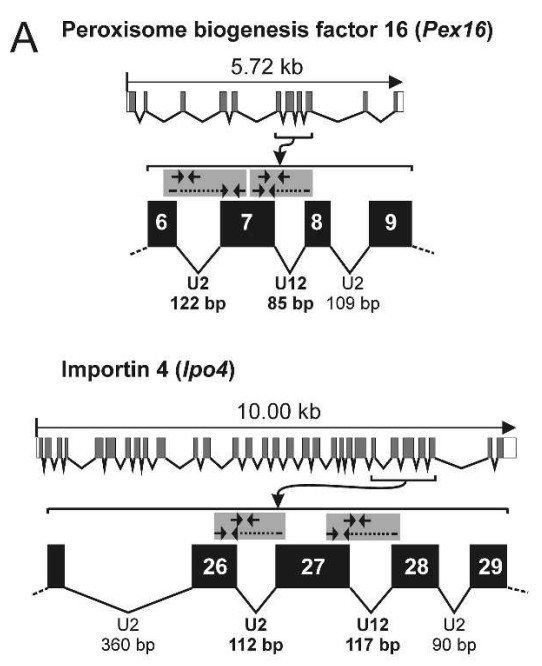

smE protein (smE)

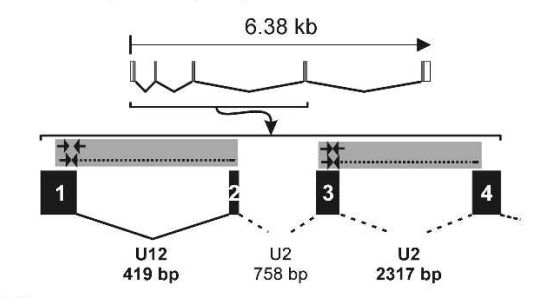

\begin{tabular}{|c|c|c|c|c|c|c|c|}
\hline \multirow[b]{2}{*}{ Gene } & \multicolumn{3}{|c|}{ U12-type introns } & \multicolumn{4}{|c|}{ U2-type introns } \\
\hline & intron & 5 ss & $3^{\prime} \mathrm{ss}$ & intron & 5 ss & PPT & 3 'ss \\
\hline Pex16 & $7-8$ & GTATCCTTAGC & ATATCCTTGACATCTTGACCGTCAG & $6-7$ & GTAAGTGGAAG & САCTGACCTTGTCTTI & TTCTAG \\
\hline Drap1 & $2-3$ & GTATCCTACTA & TTCTCCTTGAC------GTCTTCAG & $5-6$ & GTGAGTGAGGC & ACCCTGACCCCTTCCG & TCTCAG \\
\hline Ipo4 & $27-28$ & GTATCCTATGC & СATTCCTTAAC----TATGGACCAG & $26-27$ & GTGAGTGCTGT & GTGCACTCCTTCACCC & CTATAG \\
\hline Psmc4 & $9-10$ & GTATCCTTCTT & GTTTCTTTAAC-TCAGCTGCTATAG & $7-8$ & GTGAGTAGTGC & AACTGACCATGTTTCT & TCTTAG \\
\hline$s m E$ & $1-2$ & GTATCCTACGC & TTTTCCTTGAT-CGCCGTGCTGCAG & $3-4$ & GTGAGTACCCA & AAATGATTGTCATTTE & TCCCAG \\
\hline Gars & $11-12$ & ATATCСтTТCT & GATTCCTTGAC----TGTTTCATAC & $9-10$ & GTAAGGTTCTC & СTTTGCTGCTCTTCTA & GTTCAG \\
\hline \multicolumn{2}{|c|}{ Consensus } & RTATCCTTTNN & TTTTCCTTAAC------ CAG & & GTRAGTNNNNN & YYYYYYYYYYY & YYNCAG \\
\hline
\end{tabular}

FIGURE 4. Endogenous genes used in quantitative PCR (qPCR) analysis. ( $A, t o p)$ Overall exon-intron structure of the genes containing U12-type introns that were used in the analysis. Exons are indicated by boxes (gray, protein-coding region; white, untranslated region). (Bottom) Schematic illustration of the qPCR primer design. The approximate location of each qPCR primer pair used for mRNA and pre-mRNA quantifications is indicated by short arrows in gray boxes. Dotted lines indicate exon-spanning primers used to quantify mRNA levels. These primers bind to upstream and downstream exons surrounding the intron under investigation. Gene structures and intron numbering reflect the NCBI m34 assembly release (May 2005) of the mouse genome and were obtained from Ensembl database release 36 at www.ensembl.org. Intron types and sizes are indicated for selected introns. (B) Comparison of the selected U12- and U2-type introns with the published consensus sequences. The consensus sequences have been reported in Abril et al. (2005) and Burge et al. (1999). 
spliced, respectively (see Fig. 4). In addition to U12-type introns, we used a nearby U2-type intron of approximately the same size in each gene as an internal reference. Each selected U2-type reference intron contained a consensus 5 'ss sequence and a strong polypyrimidine tract, which are expected to support efficient splicing (see Fig. 4). For accurate quantification of both the U12- and U2-type introns and the spliced products, we used external concentration standards for each amplicon to compensate for possible primer-specific differences in amplification efficiency. Thus, serial dilutions of cloned genomic or cDNA fragments of each gene, resulting in a range of $10^{3}$ to $10^{7}$ molecules per amplification reaction, were included as a duplicate in each qPCR run (see Materials and Methods for details).

Our qPCR results were in stark contrast to the above results using reporter construct transfections (Fig. 2). Comparison of the U12-type intron signals from the selected genes in 3T3-D1 and L-929 cells did not reveal any significant differences in splicing efficiencies between the cell lines for five of the six genes included in our test. For the genes Drap1, Ipo4, Psmc4, Gars, and smE, the relative levels of unspliced U12-type introns varied from $<1 \%$ to $15 \%$ of the spliced mRNA level, depending on the gene, but no significant differences between the two cell lines in U2- or U12-dependent splicing levels were observed (Fig. 5C-L). We found that the level of the unspliced U12type intron was generally about two- to threefold higher than that of the U2-type introns, which is consistent with earlier results (Patel et al. 2002). The only exception was Psmc4 in L-929 cells (Fig. 5G), in which case the levels of unspliced U2- and U12-type introns were nearly equivalent. The only clear difference in splicing efficiencies between the two cell lines was detected with Pex16. In this case, the splicing of the U12-type intron was very inefficient in 3T3-D1 cells (Fig. 5B; note the different scaling of the $y$-axis in panels $A$ and $B$ as compared to the other panels in the figure) but relatively robust in L-929 cells (Fig. 5A). This inefficient splicing in 3T3-D1 cells was confirmed using independent primers specific to Pex16 exons 7 and 8 (see Fig. 4A), which allowed amplification of both mRNA and pre-mRNA in a single reaction, followed by gel analysis to verify that the correctly sized fragments were amplified. The results of this independent test consistently showed that a significant portion $(>30 \%)$ of the pre-mRNA was amplified (data not shown). Similar to the other endogenous genes we examined, the U12-type intron of Pex16 was clearly less efficiently spliced in both cell lines compared to the Pex16 U2-type intron (Fig. 5A,B).

Finally, we estimated the relative expression levels of the endogenous genes examined in Figure 5 by qPCR (Table 1). We found that the expression levels vary 30-fold among this set of genes, the expression being weakest with Pex16 and strongest with Gars. We did not observe any clear correlation between U12-type intron splicing efficiency

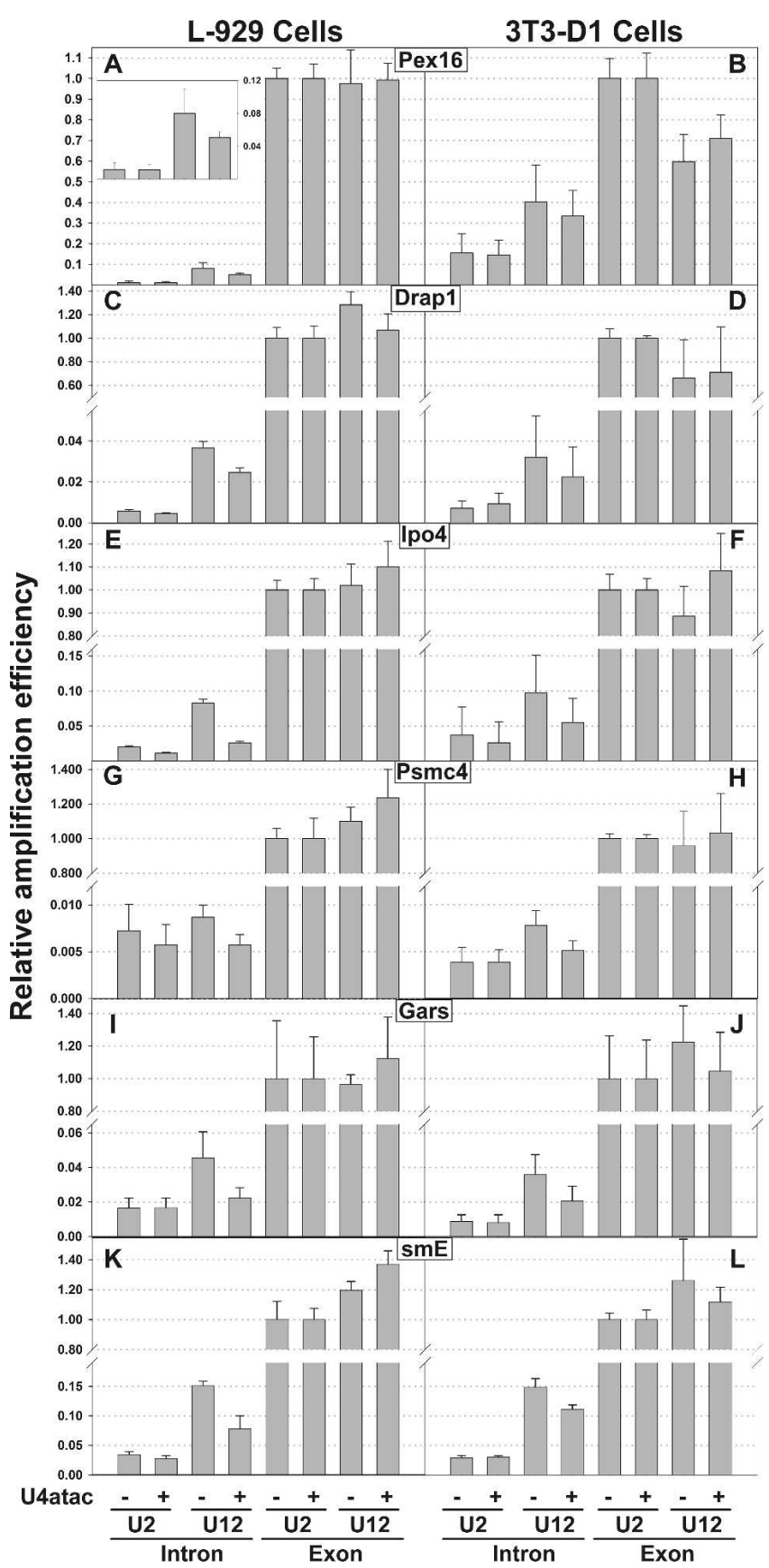

FIGURE 5. Quantitative RT-PCR analysis of the splicing of U12-type introns in endogenous genes. Stable L-929 and 3T3-D1 cell lines transfected with either a vector or a U4atac overexpression plasmid were analyzed using primers described in Figure 4. All data were normalized against the U2-type exon signal that was set to 1.0 , as described in Materials and Methods. The first four bars ("intron") in each panel represent the signal either from the unspliced U2- or U12type introns in the absence or presence of the U4atac overexpression plasmid, as indicated in the figure. The next four bars ("exon") in each panel show the corresponding data for the spliced U2- and U12type mRNAs. Note that the $y$-axis is discontinuous in the Drap1, Ipo4, Psmc4, Gars, and smE panels. Pex16 intron data for L-929 cells has been included as a scaled-up inset because of large differences between the 3T3-D1 and L-929 data. Error bars indicate standard deviation. 
TABLE 1. Relative mRNA expression levels of U12-type intron-containing genes in 3T3-D1 and L-929 cell lines

\begin{tabular}{lcc}
\hline & 3T3-D1 $^{\mathrm{a}}$ & L-929 \\
\hline Pex16 & 0.12 & 0.15 \\
Drap1 & 3.44 & 1.40 \\
Psmc4 & 0.49 & 0.79 \\
Ipo4 & 1.03 & 1.39 \\
Gars & 4.81 & 5.32 \\
smE & 1.00 & 1.00 \\
\hline
\end{tabular}

${ }^{\text {a } E x p r e s s i o n ~ l e v e l s ~ w e r e ~ d e t e r m i n e d ~ b y ~ q u a n t i t a t i v e ~ R T-P C R ~ u s i n g ~}$ primers specific to each mRNA. The obtained values were normalized to the level of $s m E$ gene that was set to 1.0.

and the expression levels of the given gene (cf. Fig. 5 and Table 1).

These results indicate that a reduction in the abundance of the U4atac/U6atac complex does not significantly affect the splicing of U12-type introns under normal conditions. In support of this conclusion, and contrary to our observations with the transient transfections with a reporter construct, the splicing of U12-type introns in 3T3-D1 cells did not improve significantly upon overexpression of U4atac snRNA; generally, the overexpression resulted in a $10 \%-50 \%$ decrease in the pre-mRNA levels, depending on the gene examined, but a similar effect was also seen with L-929 cells. Even with Pex16, which displayed very inefficient excision of the U12-type intron, the effect of U4atac snRNA overexpression yielded only an $\sim 15 \%$ improvement in splicing. Thus, we conclude that an $80 \%-$ $90 \%$ reduction in U4atac/U6atac abundance does not significantly affect the splicing of endogenous genes containing U12-type introns under normal conditions.

\section{DISCUSSION}

The lower abundance of U12-dependent spliceosome snRNP components has been suggested as a possible explanation for the observed slower excision rate of U12type introns detected both in vitro (Tarn and Steitz 1996b; $\mathrm{Wu}$ and Krainer 1996) and in vivo (Patel et al. 2002). Here we have tested this hypothesis using a mouse cell line that exhibits a fivefold reduction in the levels of U4atac snRNA. We found that the reduction in U4atac levels indeed decreases the splicing efficiency of a U12-type intron-containing reporter that has been overexpressed transiently in such cells. Coexpression of U4atac snRNA restores splicing to normal levels, suggesting that the decreased splicing efficiency was because of the reduction in U4atac levels. In contrast to the situation observed with the transiently transfected reporter gene, the reduced U4atac levels did not significantly affect the splicing of U12-type introns of the six endogenous genes we investigated. We used genes that have varying intron sizes or expression levels and found that significantly reduced levels of U4atac (and thus U4atac/U6atac) had no obvious effects on the splicing of U12-type introns. Furthermore, we found no apparent correlation between U12-type intron splicing efficiency and intron size or the expression level of the gene. Thus, our data suggest that, under normal conditions, there is a surplus of the U12-type snRNPs in the cell, and therefore their number does not limit excision of U12-type introns.

Our results of the splicing of endogenous genes containing U12-type introns agree with the conclusion of Patel et al. (2002), who found that U12-type introns are spliced more slowly than U2-type introns. Significantly, we detected slower splicing of U12-type introns with each of the six endogenous U12-type intron-containing genes investigated. Furthermore, this effect was also observed in both cell lines used in our study, thus providing further support to the suggestion by Patel et al. (2002) that the relative slowness of the observed splicing could be a general property of U12-type introns. Additionally, because we measured both mRNA and pre-mRNA levels by qPCR, we were able to estimate the splicing efficiency of each U12type intron. A comparison of unspliced versus spliced intron levels showed substantial gene-specific variation in the splicing efficiencies of the U12-type introns (Fig. 5). With the set of genes examined in this study, the variation that was consistently observed with both cell lines extended one order of magnitude. Additionally, we found one case, namely, the U12-type intron of the gene Pex16, in which the splicing was very inefficient in the U4atac-deficient 3T3-D1 cells.

The key assumption in our analysis was that the observed reduced levels of U4atac snRNA should directly impact the number of functional U4atac/U6atac complexes, and thus indirectly, the number of U12-dependent spliceosomes in the cell. If U12-type spliceosomes are rate limiting for premRNA processing, then the reduction in the U4atac number should negatively affect U12-dependent splicing efficiency in 3T3-D1 cells as compared with the control cells because the number of functional spliceosomes per cell would fall from the estimated $2 \times 10^{3}$ (Tarn and Steitz 1996a; Yu et al. 1999; data not shown) to $\sim 400$. Our data show that even with L-929 and HeLa cells, the U4atac snRNA is the limiting component for U4atac/U6atac complex maturation, as there is virtually no free U4atac snRNA in the cell (Figs. 1B, 3). Therefore, U4atac levels directly regulate the number of U4atac/U6atac complexes: The level is reduced in 3T3-D1 cells (Figs. 1B, 3) but is restored to the level observed in L-929 cells upon stable overexpression of U4atac (Fig. 3, cf. lanes 1 and 7). Consistent with these data, in L-929 cells U4atac overexpression leads to a substantial increase in U4atac/U6atac complexes (Fig. 3, lane 5). Because the U5 snRNP and the protein components of the U4atac/U6atac.U5 tri-snRNP are shared with the U2-dependent spliceosome (Tarn and Steitz 1996b; Schneider et al. 2002; Shukla and Padgett 2004), which is 100-fold more abundant, the U4atac snRNA levels are expected to directly 
influence the number of the U4atac/U6atac.U5 tri-snRNPs in the cell. U4atac and U4 snRNAs likely function analogously as essential assembly factors for the entry of U6atac or U6 into their respective spliceosomes (Yean and Lin 1991; Raghunathan and Guthrie 1998); if so, then U4atac levels should directly affect spliceosome assembly. According to the sequential spliceosome assembly model (see Brow 2002; Patel and Steitz 2003), a reduction in U4atac level would result in low tri-snRNP levels that would be expected to slow spliceosome assembly immediately after intron recognition by the U11/U12 di-snRNP, which could lead to nuclear accumulation of unspliced pre-mRNAs containing U12-type introns. However, several studies have suggested that U2-type spliceosomes may exist as preassembled holospliceosomes containing all five snRNPs (Stevens et al. 2001; Brow 2002; Nilsen 2002; however, see Tardiff and Rosbash 2006) and that the full complement of snRNPs may be needed already during the intron recognition (Malca et al. 2003). If this paradigm would be true for U12-dependent splicing, then U4atac snRNA down-regulation could also prevent the intron recognition step. Regardless of the paradigm, however, it is expected that the number of U12-dependent spliceosomes involved in premRNA processing should be reduced significantly upon U4atac down-regulation, with consequent negative effects on splicing if, indeed, the number of U12-dependent spliceosomes is rate limiting.

The fact that we observed inefficient splicing in 3T3-D1 cells with the reporter gene, but not with the endogenous genes, suggests that U12-dependent spliceosomes or the spliceosome components are not saturated by the endogenous pre-mRNAs containing U12-type introns, even when the number of spliceosomes is reduced fivefold. However, the number of spliceosomes could become limiting if cells were further challenged by overexpression of genes containing U12-type introns. Indeed, when we challenged the spliceosome with a large excess of the reporter RNA, we observed inefficient splicing of U12-type intron of the reporter construct. Because the splicing of the U12-type intron was improved by coexpressing the U4atac snRNA, the result suggests that the splicing deficiency was because of low U4atac levels. In contrast, overexpression of a reporter gene with a U12-type intron in cells containing normal levels of U4atac snRNA was not alone sufficient to saturate U12-dependent spliceosomes, as the reporter gene was spliced efficiently in 3T3-D1 cells cotransfected with the U4atac expression plasmid and in L-929 cells containing a normal amount of U4atac snRNA (Fig. 2). Similarly, a simple reduction of U4atac levels did not affect the splicing of endogenous genes, as discussed above. This result differs somewhat from the Patel et al. (2002) study, in which the overexpression of a transiently transfected reporter gene containing a U12-type intron into Drosophila S2 cells led to the accumulation of pre-mRNAs containing unspliced U12type introns. Differences in experimental systems (insect versus mammalian cell lines, transfection efficiencies, or splicing efficiencies) may explain these apparent contradictory results.

In summary, we present evidence that a fivefold reduction in the number of U12-type spliceosomes does not limit the processing of endogenous U12-type introns, suggesting that there is a surplus of U12-type spliceosomes in the cell. However, similar to the results of Patel et al. (2002), we observed slower excision of U12-type introns as compared with U2-type introns. What then is the mechanistic basis for this phenomenon? Based on the data presented here and elsewhere it is possible to rule out the spliceosome assembly steps following the first catalytic step of splicing, because both our qPCR quantifications and the experiments of Patel et al. (2002) measured pre-mRNA levels across the $5^{\prime}$ exon-5'ss junction, which requires that this junction be intact and not have undergone the first step of splicing. Even though the results from in vitro systems do not necessary reflect events in vivo, earlier in vitro studies of spliceosome complex assembly and splicing kinetics have indicated that a transition from the B-complex stage to the catalytically active $\mathrm{C}$ complex is much slower than the earlier stages of spliceosome assembly (Tarn and Steitz 1996a, b; Wu and Krainer 1996; Frilander and Steitz 1999; Frilander and Steitz 2001; Hastings and Krainer 2001). Perhaps, then, there is a regulatory modification or a rate-limiting factor working at this stage of the spliceosome assembly.

\section{MATERIALS AND METHODS}

\section{Cell culture}

The two independent isolates of the NIH-3T3 cell lines having reduced U4atac snRNA levels were initially obtained from Dr. Pekka Lappalainen (University of Helsinki, Institute of Biotechnology) and from Dr. Tomi Mäkelä (University of Helsinki, Institute of Biomedicine). L-929 (CCL-1) cells were purchased from LGC Promochem. HeLa cells were obtained from Dr. Joan Steitz (Yale University). NIH-3T3 and L-929 cells were cultured in monolayers in DMEM containing either $10 \%$ FBS or $10 \%$ horse serum, respectively. HeLa cells were cultured in suspension in RPMI containing 10\% FBS. All media were supplemented with 2 $\mathrm{mM}$ glutamine, $50 \mathrm{U} / \mathrm{mL}$ penicillin, and $50 \mu \mathrm{g} / \mathrm{mL}$ streptomycin. Transfections were performed on $35-\mathrm{mm}$ plates with FuGene 6 transfection reagent (Roche) as described by the manufacturer. Equimolar amounts of smE-CFP and U4atac overexpression construct corresponding to $0.78 \mu \mathrm{g}$ and $1.0 \mu \mathrm{g}$, respectively, were used in transient transfections. The transfection efficiencies were estimated to be $\sim 10 \%$ with both cell lines. Culture medium was supplemented with $400 \mu \mathrm{g} / \mathrm{mL}$ G418 when selecting cell lines overexpressing U4atac snRNA.

\section{Plasmids and expression vectors}

Mouse cDNAs encoding U4atac and U6atac snRNAs were initially cloned from a total RNA preparation isolated from NIH-3T3 cells by ligating a DNA oligonucleotide to the $3^{\prime}$ end of the snRNAs, followed by cDNA synthesis and PCR cloning. U4atac and U12 
genes containing endogenous promoter sequences were subcloned from genomic BAC or $\lambda$ clones, respectively. Both clones have been derived from the $129 \mathrm{~Sv}$ mouse strain. U4atac overexpression plasmid containing a U12 promoter was constructed by fusing the genomic clones described above followed by cloning into the MluI site of pEGFP-Actin (Clontech).

The smE-CFP reporter plasmid was constructed by joining the first three exons of the mouse gene $s m E$ to the cyan fluorescent protein sequence derived from the U12C plasmid (Patel et al. 2002). The $s m E$ portion of the construct was amplified by PCR from a mouse genomic DNA preparation. The insert sequence containing the smE-CFP sequence was cloned into the pC1-neo vector (Promega). All constructs were verified by sequencing.

\section{Northern blotting}

Total RNA was isolated from cells with TRIzol reagent (Invitrogen) according to manufacturer's instructions. Total RNA (2-10 $\mu \mathrm{g})$ was separated in denaturing $6 \%$ polyacrylamide gels, transferred to a nylon filter with a semi-dry blotter $(\mathrm{Owl})$ in $0.5 \times$ Trisborate-EDTA using a constant $3 \mathrm{~mA} / \mathrm{cm}^{2}$ current for $1.5-2 \mathrm{~h}$, followed by cross-linking with Stratalinker (Stratagene). For the detection of smE-CFP transcripts, $8.5 \mu \mathrm{g}$ of total RNA were separated in a $1.5 \%$ agarose gel containing formaldehyde as described by Sambrook and Russell (2001) and transferred to a nylon membrane by capillary blotting in $20 \times$ SSC overnight followed by cross-linking with Stratalinker (Stratagene).

Hybridization conditions for snRNA blots were as described (Tarn and Steitz 1996b). Radiolabeled probes were made either by random prime labeling using Rediprime (Amersham) or by in vitro transcription of the snRNA clones as described (Tarn and Steitz 1996b). U6atac snRNA was detected by a ${ }^{32} \mathrm{P}$-labeled U6atac-specific oligonucleotide as described previously (Frilander and Steitz 2001). SmE-CFP was detected by Northern hybridization using a ${ }^{32} \mathrm{P}$-labeled DNA probe.

\section{Native gel analysis and psoralen cross-linking}

Nuclear extracts, which were prepared essentially as described (Tarn and Steitz 1994), or whole-cell lysates prepared by sonication were used to detect U4atac/U6atac snRNA complexes. The snRNAs were deproteinized at low temperature $\left(37^{\circ} \mathrm{C}\right)$ in buffer containing $1 \mathrm{mg} / \mathrm{mL}$ proteinase $\mathrm{K}, 50 \mathrm{mM} \mathrm{NaCl}, 10 \mathrm{mM}$ EDTA, and $0.5 \%$ SDS for $30 \mathrm{~min}$, followed by separation in nondenaturing $8 \%$ polyacrylamide gels, transfer to nylon membrane, crosslinking, and hybridization as described above. Psoralen crosslinking of the nuclear extract preparations was done essentially as described by Frilander and Steitz (2001).

\section{RT-PCR analysis}

RNAs were extracted with TRIzol according to the manufacturer's instructions, except that additional acidic phenol $(\mathrm{pH} 5.0)$ and chloroform extraction steps were included prior to the RNA precipitation step. RNA samples used in real-time PCR were also treated with DNase (DNA-free, Ambion) to remove traces of contaminating genomic DNA. Reverse transcription was then performed using SuperScript III (Invitrogen) and oligo- $\mathrm{dT}_{20}$ primer or, in some cases, with random hexamer primers.

Quantitative RT-PCR reactions were performed with ABI PRISM 7000 Sequence Detection System in a $20 \mu \mathrm{L}$ volume that contained $10 \mu \mathrm{L}$ of ABsolute SYBR Green ROX Mix (ABgene), $0.175 \mu \mathrm{M}$ of each primer, and cDNA equivalent to $125 \mathrm{ng}$ of total RNA. PCR conditions were $50^{\circ} \mathrm{C}$ for $2 \mathrm{~min}, 95^{\circ} \mathrm{C}$ for $15 \mathrm{~min}$, followed by 37 cycles of $95^{\circ} \mathrm{C}$ for $15 \mathrm{sec}$ and $60^{\circ} \mathrm{C}$ for $1 \mathrm{~min}$. In each run, the experimental samples were included in triplicate and DNA concentration standards (see below) in duplicate. At least three independent runs were performed for each gene. The homogeneity of each PCR product was confirmed by melting curve analysis as the final step in the quantitative PCR program. Negative controls containing no template or lacking the cDNA synthesis step were also included in each run. Absolute concentrations for each amplicon were obtained from parallel concentration standards that were included for each amplified fragment in each run. The concentration standards were serial dilutions of cloned genomic (intron-containing) and cDNA (spliced) fragments of known concentration for each gene investigated. The range of serial dilutions used for the concentration standards covered $10^{3}$ to $10^{7}$ molecules. The concentrations of the DNAs used for standard curves were initially quantified by a diode-array spectrophotometer (Hewlett Packard 8452A) and further confirmed by analysis in agarose gels followed by quantification with a CCD-camera system (Fuji LAS-3000). For each acceptable realtime PCR run, the correlation coefficient $\left(R^{2}\right)$ of the concentration standards was $>0.97$. The results were analyzed with ABI PRISM 7000 SDS 1.1 software. The signals for the U2- and U12type introns and the spliced U12-type mRNA from individual real-time PCR runs were normalized against the spliced U2-type mRNA signal that was set to 1.0.

\section{ACKNOWLEDGMENTS}

We thank Joan Steitz and Abhijit Patel for the U12C reporter plasmid and Oscar Puig and the members of the Frilander laboratory for excellent comments during the work. Oscar Puig and the members of the Frilander laboratory are thanked for critical reading of the manuscript. Janne Turunen is thanked for sharing unpublished data on Ipo4 in vitro splicing and complex assembly. This work was supported by the Academy of Finland (Grants 50116 and 50527 to M.J.F.) and the Sigrid Juselius Foundation. H.K.J.P. was supported by the Viikki Graduate School in Biosciences.

Received July 3, 2006; accepted July 25, 2006.

\section{REFERENCES}

Abril, J.F., Castelo, R., and Guigo, R. 2005. Comparison of splice sites in mammals and chicken. Genome Res. 15: 111-119.

Brow, D.A. 2002. Allosteric cascade of spliceosome activation. Annu. Rev. Genet. 36: 333-360.

Burge, C.B., Padgett, R.A., and Sharp, P.A. 1998. Evolutionary fates and origins of U12-type introns. Mol. Cell 2: 773-785.

Burge, C.B., Tuschl, T., and Sharp, P.A. 1999. Splicing of precursors to mRNAs by the spliceosome. In The RNA world (eds. R.F. Gesteland et al.), pp. 525-560. Cold Spring Harbor Laboratory Press, Cold Spring Harbor, NY.

Frilander, M.J. and Steitz, J.A. 1999. Initial recognition of U12dependent introns requires both U11/5' splice-site and U12/ branchpoint interactions. Genes \& Dev. 13: 851-863.

- 2001. Dynamic exchanges of RNA interactions leading to catalytic core formation in the U12-dependent spliceosome. Mol. Cell 7: 217-226. 
Hall, S.L. and Padgett, R.A. 1996. Requirement of U12 snRNA for in vivo splicing of a minor class of eukaryotic nuclear pre-mRNA introns. Science 271: 1716-1718.

Hastings, M.L. and Krainer, A.R. 2001. Functions of SR proteins in the U12-dependent AT-AC pre-mRNA splicing pathway. RNA 7: $471-482$.

Incorvaia, R. and Padgett, R.A. 1998. Base pairing with U6atac snRNA is required for $5^{\prime}$ splice site activation of U12-dependent introns in vivo. RNA 4: 709-718.

Kolossova, I. and Padgett, R.A. 1997. U11 snRNA interacts in vivo with the $5^{\prime}$ splice site of U12-dependent (AU-AC) pre-mRNA introns. RNA 3: 227-233.

Levine, A. and Durbin, R. 2001. A computational scan for U12dependent introns in the human genome sequence. Nucleic Acids Res. 29: 4006-4013.

Malca, H., Shomron, N., and Ast, G. 2003. The U1 snRNP base pairs with the $5^{\prime}$ splice site within a penta-snRNP complex. Mol. Cell. Biol. 23: 3442-3455.

Montzka, K.A. and Steitz, J.A. 1988. Additional low-abundance human small nuclear ribonucleoproteins: U11, U12 etc. Proc. Natl. Acad. Sci. 85: 8885-8889.

Nilsen, T.W. 2002. The spliceosome: No assembly required? Mol. Cell 9: 8 -9.

Patel, A.A. and Steitz, J.A. 2003. Splicing double: Insights from the second spliceosome. Nat. Rev. Mol. Cell Biol. 4: 960-970.

Patel, A.A., McCarthy, M., and Steitz, J.A. 2002. The splicing of U12type introns can be a rate-limiting step in gene expression. EMBO J. 21: 3804-3815.

Raghunathan, P.L. and Guthrie, C. 1998. A spliceosomal recycling factor that reanneals $\mathrm{U} 4$ and $\mathrm{U} 6$ small nuclear ribonucleoprotein particles. Science 279: 857-860.

Sambrook, J. and Russell, D.W. 2001. Molecular cloning: A laboratory manual. Cold Spring Harbor Laboratory Press, Cold Spring Harbor, New York.

Santoro, B., De Gregorio, E., Caffarelli, E., and Bozzoni, I. 1994. RNA-protein interactions in the nuclei of Xenopus oocytes: Complex formation and processing activity on the regulatory intron of ribosomal protein gene L1. Mol. Cell. Biol. 14: 6975-6982.

Schneider, C., Will, C.L., Makarova, O.V., Makarov, E.M., and Lührmann, R. 2002. Human U4/U6.U5 and U4atac/U6atac.U5 Tri-snRNPs exhibit similar protein compositions. Mol. Cell. Biol. 22: 3219-3229.

Schneider, C., Will, C.L., Brosius, J., Frilander, M.J., and Lührmann, R. 2004. Identification of an evolutionarily divergent
U11 small nuclear ribonucleoprotein particle in Drosophila. Proc. Natl. Acad. Sci. 101: 9584-9589.

Shukla, G.C. and Padgett, R.A. 1999. Conservation of functional features of U6atac and U12 snRNAs between vertebrates and higher plants. RNA 5: 525-538.

. 2004. U4 small nuclear RNA can function in both the major and minor spliceosomes. Proc. Natl. Acad. Sci. 101: 93-98.

Stevens, S.W., Barta, I., Ge, H.Y., Moore, R.E., Young, M.K., Lee, T.D., and Abelson, J. 2001. Biochemical and genetic analyses of the U5, U6, and U4/U6.U5 small nuclear ribonucleoproteins from Saccharomyces cerevisiae. RNA 7: 1543-1553.

Tardiff, D.F. and Rosbash, M. 2006. Arrested yeast splicing complexes indicate stepwise snRNP recruitment during in vivo spliceosome assembly. RNA 12: 968-979.

Tarn, W.-Y. and Steitz, J.A. 1994. SR proteins can compensate for the loss of U1 snRNP functions in vitro. Genes \& Dev. 8: 2704-2717. . 1996a. Highly divergent U4 and U6 small nuclear RNAs required for splicing rare AT-AC introns. Science 273: 1824-1832.

. 1996b. A novel spliceosome containing U11, U12 and U5 snRNPs excises a minor class (AT-AC) intron in vitro. Cell 84: 801-811.

1997. Pre-mRNA splicing: The discovery of a new spliceosome doubles the challenge. Trends Biochem. Sci. 22: 132-137.

Will, C.L. and Lührmann, R. 2005. Splicing of a rare class of introns by the U12-dependent spliceosome. Biol. Chem. 386: 713-724.

Will, C.L., Schneider, C., Reed, R., and Lührmann, R. 1999. Identification of both shared and distinct proteins in the major and minor spliceosomes. Science 284: 2003-2005.

Will, C.L., Schneider, C., Hossbach, M., Urlaub, H., Rauhut, R., Elbashir, S., Tuschl, T., and Lührmann, R. 2004. The human 18S U11/U12 snRNP contains a set of novel proteins not found in the U2-dependent spliceosome. RNA 10: 929-941.

$\mathrm{Wu}, \mathrm{Q}$. and Krainer, A.R. 1996. U1-mediated exon definition interactions between AT-AC and GT-AG introns. Science 274: $1005-1008$.

Yean, S.L. and Lin, R.J. 1991. U4 small nuclear RNA dissociates from a yeast spliceosome and does not participate in the subsequent splicing reaction. Mol. Cell. Biol. 11: 5571-5577.

Yu, Y.-T., Scharl, E., Smith, C.M., and Steitz, J.A. 1999. The growing world of small nuclear ribonucleoproteins. In The RNA world (eds. R.F. Gesteland et al.). Cold Spring Harbor Laboratory Press, Cold Spring Harbor, NY.

Zhu, W. and Brendel, V. 2003. Identification, characterization and molecular phylogeny of U12-dependent introns in the Arabidopsis thaliana genome. Nucleic Acids Res. 31: 4561-4572. 

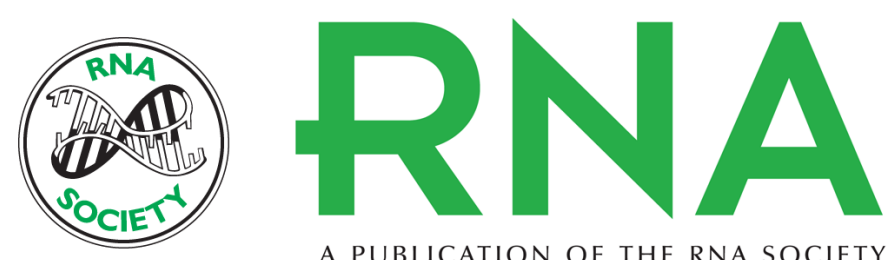

A PUBLICATION OF THE RNA SOCIETY

\section{The abundance of the spliceosomal snRNPs is not limiting the splicing of U12-type introns}

Heli K.J. Pessa, Annukka Ruokolainen and Mikko J. Frilander

RNA 2006 12: 1883-1892

References This article cites 33 articles, 22 of which can be accessed free at:

http://rnajournal.cshlp.org/content/12/10/1883.full.html\#ref-list-1

License

Email Alerting Receive free email alerts when new articles cite this article - sign up in the box at the Service top right corner of the article or click here.

To subscribe to RNA go to:

http://rnajournal.cshlp.org/subscriptions 